\title{
Pedunculopontine Tegmental Nucleus Controls Conditioned Responses of Midbrain Dopamine Neurons in Behaving Rats
}

\author{
Wei-Xing Pan and Brian I. Hyland \\ Department of Physiology, School of Medical Sciences, University of Otago, Dunedin 9001, New Zealand
}

\begin{abstract}
Midbrain dopamine (DA) neurons respond to sensory cues that predict reward. We tested the hypothesis that projections from the pedunculopontine tegmental nucleus (PPTg) are involved in driving this DA cell activity. First, the activity of PPTg and DA neurons was compared in a cued-reward associative learning paradigm. The majority of PPTg neurons showed phasic responses to the onset of sensory cues, at significantly shorter latency than DA cells, consistent with a PPTg-to-DA transmission of information. However, unlike DA cells, PPTg responses were almost entirely independent of whether signals were associated with rewards. Second, DA neuron responses to the cues were recorded in free-moving rats during reversible inactivation of the PPTg by microinfusion of local anesthetic. The results showed clear suppression of conditioned sensory responses of DA neurons after PPTg inactivation that was not seen after saline infusion or in non-DA cells. We propose that the PPTg relays information about the precise timing of attended sensory events, which is integrated with information about reward context by DA neurons.
\end{abstract}

Key words: classical conditioning; reward; associative learning; single-unit recording; conscious animal; dopamine

\section{Introduction}

Midbrain dopamine (DA) cells play a central role in reward processing (Blackburn et al., 1992; Wise, 2002), and at the network level, these neurons have been postulated to provide a reward prediction error signal vital for appetitive conditioning (Schultz, 1998). DA cells in monkeys and rats respond at relatively short latency to conditioned stimuli in classical and operant appetitive conditioning tasks (Miller et al., 1981; Schultz, 1998; Hyland et al., 2002; Fiorillo et al., 2003) and show several classical phenomena of conditioned behavior such as generalization and blocking (Mirenowicz and Schultz, 1996; Waelti et al., 2001). However, whereas the output of DA cells under these conditions is well characterized, the information processing performed by DA neurons remains unclear because of a lack of information about the nature of the inputs they receive.

DA cells receive excitatory and inhibitory inputs from several descending and ascending projections that might play different roles in activating or inhibiting DA cell activity (Diana and Tepper, 2002). Of these, the pedunculopontine tegmental nucleus (PPTg) is increasingly recognized as potentially having a key role in regulating conditioned responses of DA cells (Schultz, 1998; Brown et al., 1999). The PPTg contains cholinergic and glutamatergic cells that project to the ventral tegmental area (VTA) and the substantia nigra (Beninato and Spencer, 1987; Bolam et al., 1991; Futami et al., 1995). Stimulation of these afferents activates midbrain DA cells with short latency (Scarnati et al., 1984; Lok-

Received Jan. 19, 2005; revised March 13, 2005; accepted April 6, 2005

This research was supported by grants from the New Zealand Neurological Foundation and the New Zealand Lottery Grants Board.

Correspondence should be addressed to Brian Hyland, Department of Physiology, School of Medical Sciences, P.O. Box 913, Dunedin 9001, New Zealand. E-mail: brian.hyland@otago.ac.nz.

D0I:10.1523/JNEUROSCI.0277-05.2005

Copyright $\odot 2005$ Society for Neuroscience $\quad$ 0270-6474/05/254725-08\$15.00/0 wan et al., 1999) and evokes DA release in the areas of dopaminergic innervation (Forster and Blaha, 2003).

At the behavioral level, lesions of PPTg impair attention (Inglis et al., 2001) and the learning of associations between a conditioned stimulus and a primary reward (Inglis et al., 1994, 2000). Furthermore, some evidence from recordings in cats suggest that PPTg neuronal responses to auditory stimuli may be context dependent (Dormont et al., 1998), indicating a degree of plasticity similar to that seen in DA cell responses to predicted rewards (Schultz, 1998). However, direct comparisons of PPTg and DA cell activity have not been performed in the same task in behaving animals, and several questions remain about the comparative activity of neurons in the two structures. For instance, is the pattern of PPTg responses consistent with that seen in DA cells, are PPTg responses reward dependent, and is the relative latency of these responses compatible with PPTg-to-DA transmission of information? Furthermore, there is no direct evidence available about whether integrity of PPTg function is necessary for normal responses of DA cells to conditioned signals.

In the present study, we investigated in conscious rats the role of PPTg in regulating DA cell responses to conditioned stimuli. First, we determined the latency, pattern, and reward-dependent plasticity of PPTg responses in a classical conditioning paradigm and compared these data with DA cell activity. Second, we recorded DA cell activity in the same behavioral paradigm during inactivation of PPTg to determine whether inputs from PPTg are necessary for DA cell responses.

\section{Materials and Methods}

Animals and surgery. This study was approved by the University of Otago Animal Ethics Committee. Twenty-two male Wistar rats weighing $300-$ $400 \mathrm{~g}$ were used. They were initially housed with ad libitum access to food and water in a temperature- and humidity-controlled room maintained 
on a $12 \mathrm{~h}$ light/dark cycle. A 30 gauge stainless-steel cannula containing a bundle of 10 microwire recording electrodes was stereotaxically implanted under sodium pentobarbital $(60 \mathrm{mg} / \mathrm{kg}$, i.p.) anesthesia with additional local anesthetic and systemic antibiotic cover using a sterile technique. In the PPTg recording group, the electrode tips were left just above the PPTg area $(7.8 \mathrm{~mm}$ posterior to bregma, $2.0 \mathrm{~mm}$ lateral to midline, and $6.5 \mathrm{~mm}$ below the surface of skull). In the PPTg inactivation/DA cell recording group, the electrode tips were left just above the $\mathrm{VTA} /$ medial substantia nigra pars compacta $(\mathrm{SNc})(5.3 \mathrm{~mm}$ posterior to bregma, $1.0 \mathrm{~mm}$ lateral to midline, and $7.5 \mathrm{~mm}$ below the skull surface). The microelectrode cannula was mounted on an on-head microdrive, which allowed advance of the electrodes up to $3 \mathrm{~mm}$ during the subsequent recording sessions. The electrode wires were connected to male amphenol pins mounted in a McIntyre socket (Science Technology Centre, Ottawa, Ontario, Canada). The electrode assembly was affixed to the skull using six stainless-steel screws and dental acrylic.

For PPTg inactivation experiments, a 24 gauge stainless-steel guide cannula was implanted into the PPTg ipsilateral to the DA recording electrodes. An obturator was inserted into the guide cannula to prevent occlusion. All animals were allowed 1 week to recover from surgery before any recordings.

Electrophysiological methods. The recordings were performed in a clear Perspex box in a darkened room. During recording, the electrode leads in the plug on the animal's head were connected to the recording apparatus via a flexible cable that incorporated impedance-matching unity-gain amplifiers, suspended from a low-noise electrical swivel. Signals from the electrodes were amplified $(2-10,000 \times)$, filtered $(0.5-10 \mathrm{kHz}$ bandpass), and monitored on oscilloscopes. Any channels with cellular activity were digitized $(20 \mathrm{kHz})$ and recorded on computer using Discovery software (DataWave Technologies, Longmont, CO). The extracellularly recorded action potentials were discriminated from each other and from noise using multiple features of the wave shape. When recording from midbrain DA cell fields, dopaminergic neurons were identified by firing rate $<10 \mathrm{~Hz}$ and profound $(>50 \%)$ suppression in firing rate by the $\mathrm{D}_{2}$ agonist apomorphine (750 $\mu \mathrm{g} / \mathrm{kg}$, i.p.) (Bunney et al., 1973; Aghajanian and Bunney, 1977; Grace and Bunney, 1980, 1983; Aebischer and Schultz, 1984). These cells included all those with relatively long duration and complex waveforms, but for initial screening, $1 \mathrm{~ms}$ was set as the minimum action potential duration for DA neurons. This is narrower than described in classic studies in reduced preparations, because of the use of high-pass filtering to stabilize the baseline of recordings obtained from freely moving animals. Durations of $\sim 1 \mathrm{~ms}$ represent the typical cutoff between DA and slow-firing non-DA cells under these recording conditions in both rats and monkeys (Schultz, 1986; Romo and Schultz, 1990; Ljungberg et al., 1992; Hyland et al., 2002).

Recording procedures. Rats were placed on a fluid-restriction regimen by limiting access to water to that obtained during recording sessions plus 10 min per day ad libitum access with $2 \mathrm{~d}$ free of restriction per week. Animals were monitored with daily weighing. Thirsty rats were trained to get small volumes of sweet water $(0.005 \mathrm{M}$ saccharin solution; $0.05 \mathrm{ml}$ per trial), which were delivered to a spout from the reservoir by briefly releasing a solenoid valve (Med Associates, Georgia, VT). Licking at the spout was detected by the tongue breaking an infrared beam across the spout opening.

In the PPTg recording group, encountered cells were recorded for blocks of trials in up to four different paradigms. (1) In "random reward," the solenoid delivering the fluid rewards was activated at pseudorandom intertrial intervals. The random intertrial interval began once the previous reward had been retrieved. This paradigm examined whether the cell was activated by the click sound made by the solenoid at reward delivery or in association with licking. (2) In "signal only," 100 stimuli ( $\sim 50$ stimuli of each tone or light) were delivered at pseudorandom intervals and in random order, and during this time, no liquid was delivered. This paradigm examined whether the cell responded to arbitrary sensory stimuli. The sensory signals were $0.5 \mathrm{~s}$ in duration, and were delivered from a speaker ( $4.5 \mathrm{kHz}$; SonAlert; Med Associates) and a $3 \mathrm{~W}$ bulb mounted above the drinking spout. (3) Conditioning of cell responses was tested with "signaled reward". In this condition, the tone or light was paired with reward delivery. The reward fluid was given either immediately at the end of the cue or, in some tests, with a $1 \mathrm{~s}$ delay. Tone-reward and light-reward pairings were mixed randomly in a session of 100 trials. Behavioral conditioning was evidenced by increased licking after cue onset and before reward delivery. Conditioning of the cell was indicated by the development, or alterations in amplitude or sign, of firing rate modulations time locked to the cues.

In the PPTg inactivation study, rats were exposed to the signaled reward paradigm before recordings to ensure that any conditioned responses to these cues had developed in VTA DA cells. For each recorded cell, a period of baseline data was obtained in the signaled reward task. A needle attached by flexible tubing to a $10 \mu \mathrm{l}$ Hamilton syringe was then inserted into the cannula, and either $0.5 \mu \mathrm{l}$ of $1 \%$ lidocaine hydrochloride or the same volume of saline was infused into the PPTg at the rate of $0.1 \mu \mathrm{l} / \mathrm{min}$ using a manual syringe microdrive. Recording was then continued for at least $30 \mathrm{~min}$ (100 trials). The rat was then allowed to rest for 20-30 $\mathrm{min}$, then either saline or lidocaine was infused, and recordings continued. The order of testing was alternated in different DA cells.

Histology. The recording locations were also confirmed by postmortem histology. After completion of the experiments, lesions were produced at the tip of wires from which recordings were made by passing DC ( $9 \mathrm{~V}$ for $1-2 \mathrm{~min}$ ). After $2 \mathrm{~d}$ of survival time, rats were killed by anesthetic overdose and perfused with saline and then formalin solution, the brains were sectioned on a freezing microtome, and the position of the marking lesions were determined.

Data analyses. Changes in firing rate associated with the various task events were examined by constructing averaged peristimulus time histograms (PSTHs) and trial-by-trial dot raster displays. For PPTg cells, bin widths of $5 \mathrm{~ms}$ were used. For slower-firing DA cells, bin widths of $25 \mathrm{~ms}$ were required. Response onset latencies were measured from the left edge of the first bin after a stimulus to cross a threshold set at +2 SD of the baseline mean firing rate, calculated from the $2 \mathrm{~s}$ before the first stimulus. To quantify changes in DA cell responses to cues after infusions into the PPTg, mean firing rate was calculated within the epoch 50-200 ms from the onset of each stimulus (light cue, tone cue, or solenoid). Solenoid responses were analyzed separately for trials in which the solenoid followed tone and light cues. Data were entered into a two (treatment, saline or lidocaine) by four (stimulus) ANOVA with repeated measures (General Linear Model; SPSS statistical software, version 11; SPSS, Chicago, IL)

\section{Results}

\section{Experiment 1. Characteristics of PPTg neuron activity}

One hundred and fifty-four PPTg cells were recorded in 10 rats. All recording procedures were completed in 140 of these cells. Histology showed that all of the electrode tracks on which these cells were recorded were located in the region of the PPTg (Fig. $1 A$ ). The firing rate of these cells ranged from 0.5 to $60 \mathrm{~Hz}$ (average, $13.6 \mathrm{~Hz})$.

Almost all $(86 \% ; 120$ of 140) PPTg cells responded to auditory and/or visual stimuli before they were paired with reward (Fig. 2). Four of these cells also showed modulation of activity in association with licking movements. The remaining 14\% (20 of 140) cells did not respond to either tone or light before conditioning, although four showed lick-related activity, and these cells did not develop a new response after conditioning. Thus, PPTg cell responses to sensory events can occur independent of any association with reward. We did find two cells with responses that could be interpreted as being the result of conditioning. These cells responded to the cues with a slight inhibitory pause when the cues were presented alone, but after the cues were paired with reward delivery, the response changed to a clear excitation. However, all remaining cells showed no sign of habituation or extinction nor changes in sign or amplitude of responses when the cues were paired with reward delivery.

As shown by the examples in Figure 2, PPTg cells responded to both tone and light stimuli. However, responses to tone stimuli were more common. Overall, 98\% (117 of 120) of affected cells 




Figure 1. Location of recording and infusion sites $A$, PPTg recording experiments. The vertical lines show reconstructed positions of recording-electrode tracks in PPTg mapped on standard atlas sections (Paxinos and Watson, 1997). The boundary of PPTg is indicated by the heavy line on atlas sections. The arrow on the example Niss--stained histological section indicates the electrode track within the PPTg region (oval). Scale bar, $1 \mathrm{~mm}$ (for all sections). B, PPTg inactivation/DA cell recording experiments. Top, The filled circles on atlas sections and the arrow on the example histological section show the position of tips of the infusion cannulas in PPTg. Bottom, The vertical lines on the atlas section and the arrow on the histological section show the position of recording-electrode tracks in VTA/medial SNc. AP, Anteroposterior coordinate (in millimeters) relative to bregma.

responded to tone, with 53\% (63 of 120) being tone selective and $45 \%$ (54 of 120) nonselectively responding to tone or light. In contrast, the light cue influenced $48 \%$ (57 of 120) of the cells, the majority of which were nonselective, with only $2.5 \%$ (3 of 120) responding exclusively to light.

PPTg cell responses to the cues showed a rich variety of patterns. Figure 3 shows examples of the response types and their prevalence. Pooled across stimulus modality, the most common response type (38\% of all responses) was a phasic increase in firing after stimulus onset, producing a discrete peak in the PSTH. As Figure 3 shows, there were also neurons that changed firing rate throughout the stimulus and a proportion of responses specifically to stimulus offset. Considering the light and auditory stimuli separately, there were some interesting differences. Most notably, responses to light were dominated by exclusive stimulus-offset excitations (39\% of all responses), a type that was never seen in response to tone. On the other hand, responses that included a tonic component were much more likely to be encountered after tone than light stimuli (31 and $4 \%$ of responses, respectively). The significance of these differences remains obscure, but they provide evidence for different sources of afferent input carrying modality-specific information to the PPTg.
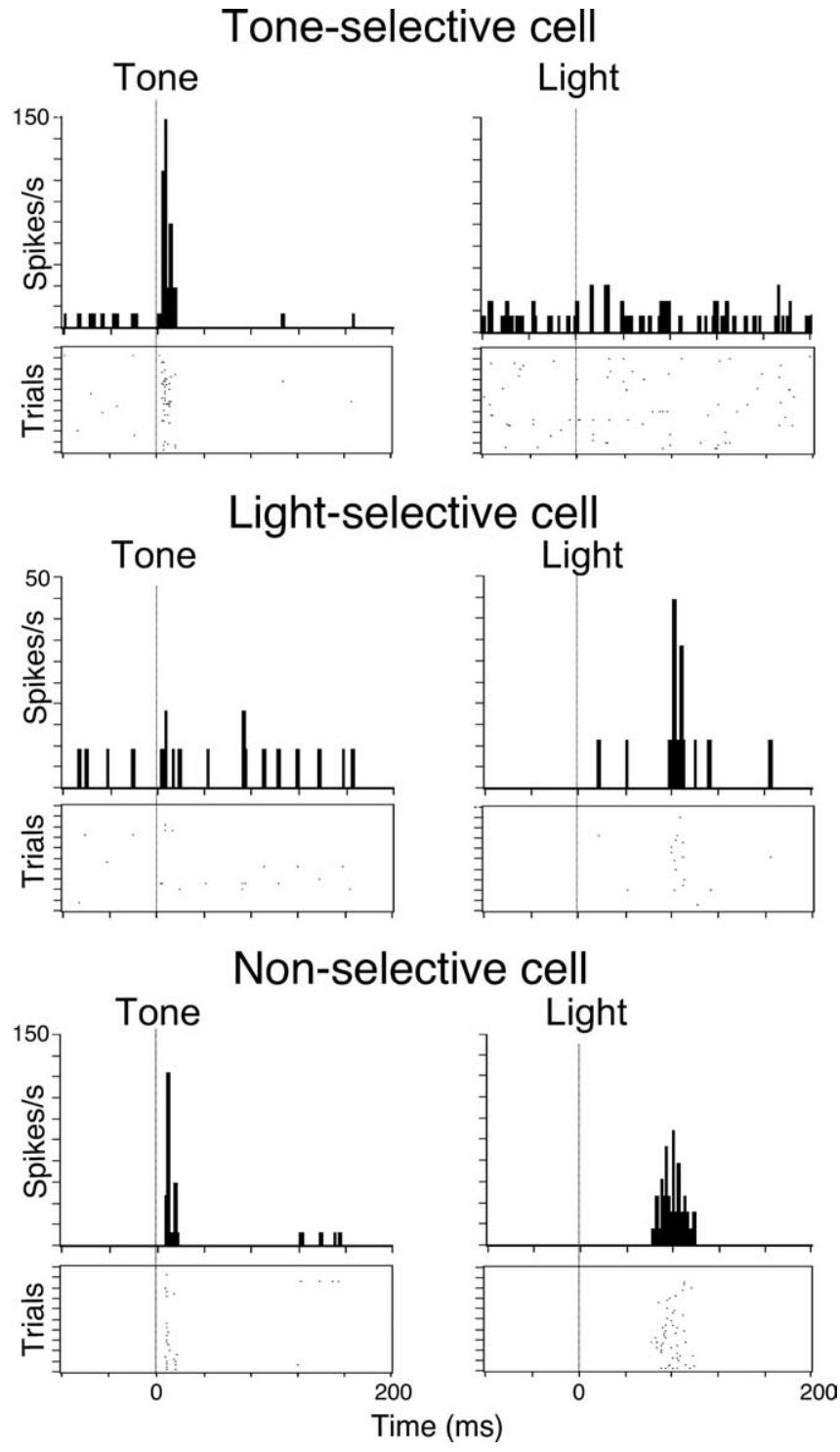

Figure 2. Example PPTg cell responses to light and tone cues. $\boldsymbol{A}$, Tone-selective cell. The histogram and dot raster show average firing rate and individual action potentials, respectively, for trials aligned to onset (time 0 ) of tone stimulus (left PSTH) or light stimulus (right PSTH). The analyses were separated from a single block of cues-only paradigm (no rewards delivered), in which the two stimuli were randomly intermingled. B, A light-selective cell. C, A nonselective cell.

As illustrated by the example cells in Figure 2, a prominent feature of PPTg responses was the short latency of excitatory responses, in particular to tones. Additional examples and cumulative frequency distribution analyses of latency of excitatory responses are provided in Figure 4. Tone latencies for excitations ranged from 5 to $35 \mathrm{~ms}$ with most $<10 \mathrm{~ms}$ and a mean of $7.7 \pm$ $0.5 \mathrm{~ms}$ (mean \pm SEM; $n=113$ ). The few initial inhibitory responses were less consistent and longer in latency $(32.5 \pm 10.3$ $\mathrm{ms} ; n=4)$. Excitatory responses to light were overall longer in latency $(72.7 \pm 5.1 \mathrm{~ms} ; n=29)$, and in this modality, latencies of inhibitions $(75.0 \pm 8.6 \mathrm{~ms} ; n=7)$ were similar to those for excitations.

\section{Experiment 2. Effect of PPTg inactivation on DA cell activity} To investigate whether the processing of sensory information in PPTg demonstrated in the previous section has a functional im- 
pact on DA cell activity, we recorded from ventral midbrain DA neurons, before and after inactivation of the ipsilateral PPTg by infusion of lidocaine through a chronically implanted infusion cannula. Recording and infusion positions were confirmed in postmortem histology (Fig. $1 B$ ). These experiments were performed while the rats retrieved fluid rewards in the same cuereward associative learning task used to investigate the PPTg responses in experiment 1.

Sixty-five cells were recorded from $\mathrm{VTA} /$ medial SNc in seven rats prepared with PPTg cannulas, of which seven cells met the criteria for classification as dopaminergic. All of the DA cells (mean firing rate, $4.6 \pm 2.8 \mathrm{~Hz}$ ) showed excitatory responses to conditioned stimuli before PPTg infusion, including tone and light cue signals and the click sound of the solenoid that controlled delivery of fluid reward. Five of the dopamine cells responded to both auditory and visual cues, one was selective for auditory signals, responding only to the solenoid and the auditory cue signal, and one was light specific, not responding at all to the auditory cue or solenoid click, although this was the most proximate predictor of reward. The latency of these responses was longer than for PPTg cells recorded in the same paradigm. Figure $4 A$ shows the responses of a DA cell to tone and light cues for comparison with a single PPTg cell from experiment 1 . The DA cell activation is clearly later than in the PPTg cell and less tightly coupled to the time of stimulus onset, resulting in a much more diffuse and broader peak, an effect that is exaggerated by the timescale required to visualize the very narrow and early PPTg response. As indicated in Figure $4 B$, over all the cells, there was little overlap in the distribution of latencies for PPTg and DA cells, and the average DA cell latencies to tone ( $65 \pm 3.4$ $\mathrm{ms} ; n=6)$ and light ( $120 \pm 2.6 \mathrm{~ms} ; n=6)$ were significantly longer than for the PPTg cells ( $p<0.01$ for both comparisons; Student's $t$ test).

Infusion of lidocaine into the PPTg resulted in a significant and reversible reduction of response amplitudes of all seven DA cells. Figure $5 A$ shows the response to light cues of the light-selective DA cell, tracked over the duration of a PPTg inactivation experiment. The response to the cue was reduced in amplitude for a period after the infusion and then recovered. Interestingly, unilateral inactivation of the PPTg did not affect the task performance in these well trained rats, which continued to lick at the spout after the infusion. Thus,

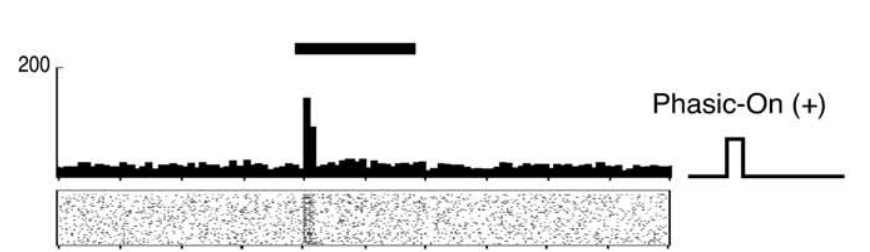

Tone Light

$84 \% \quad 41 \%$

$(117 / 140)$

$(57 / 140)$

$48 \%$

$3.5 \%$

$(56 / 117)$

$(2 / 57)$
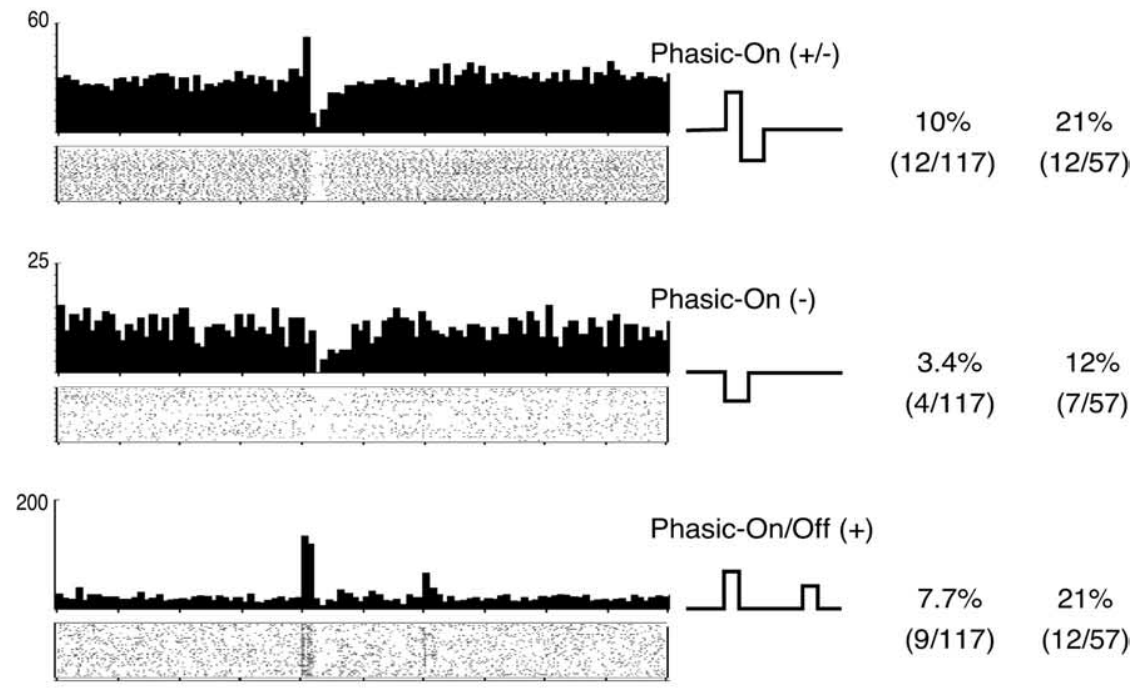

100

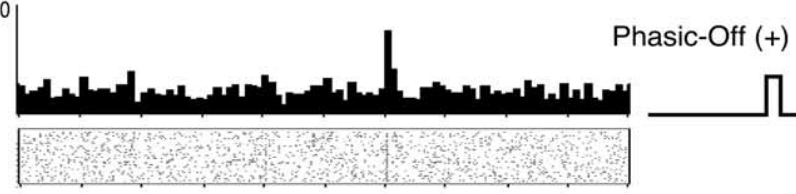

$0 \%$

$39 \%$

$(0 / 117)$

(22/57)
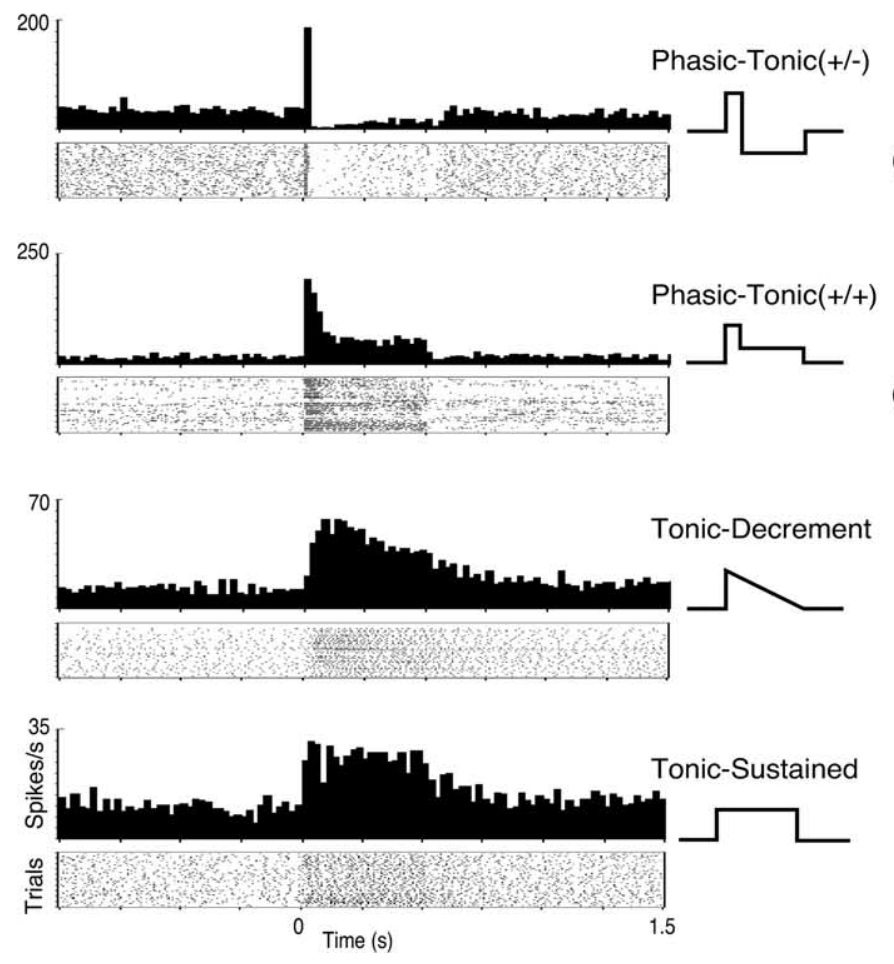

Figure 3. Response profiles of PPTg cells to tone and light stimuli. The histograms and dot rasters show average firing rate and individual action potentials, respectively, aligned to stimulus onset for examples of each response type encountered. The black bar shows duration ( $0.5 \mathrm{~s}$ ) of stimulus (either tone or light). Responses are classified as being phasic or tonic, occurring at onset $(0 \mathrm{n})$ or offset ( $0 \mathrm{ff}$ ) of stimulus, and as excitatory (+) or inhibitory $(-)$. The proportions of cells with each profile are given on the right, separately for tone and light stimuli. 

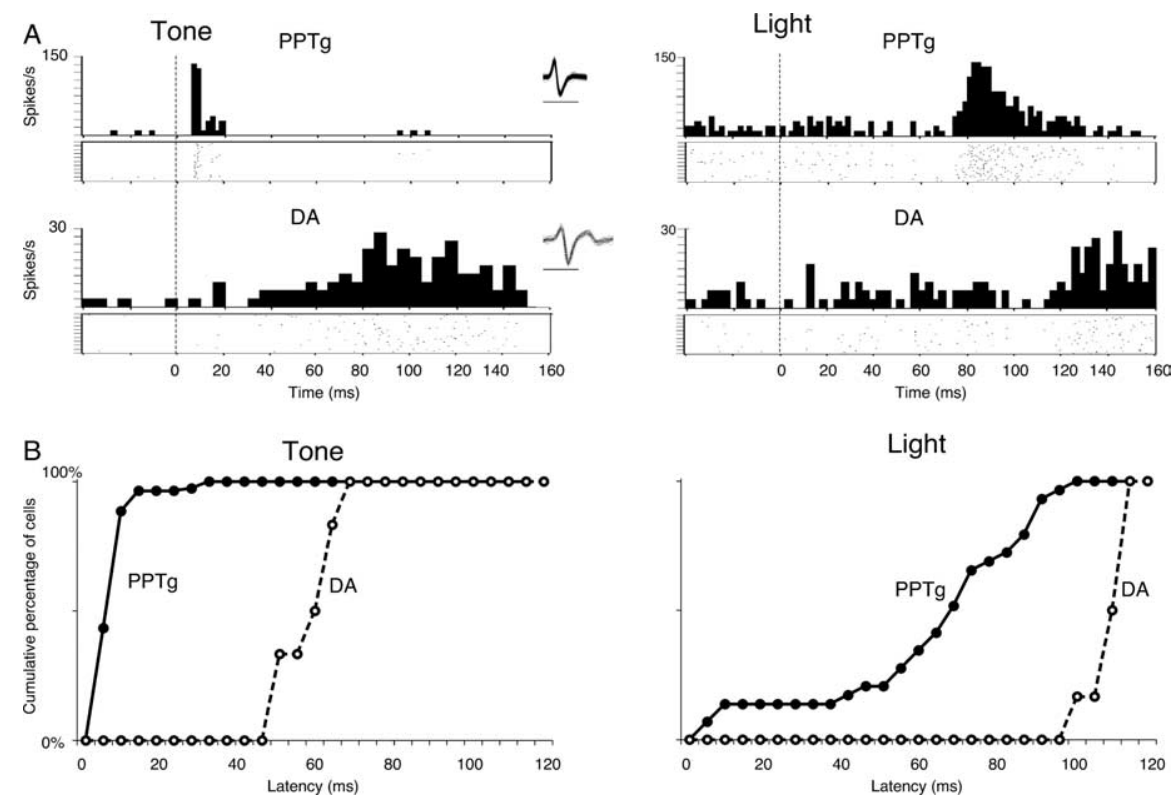

Figure 4. Comparison of latency for tone and light responses and between PPTg and DA cells. $A$, Example responses to tone and light stimuli. The histograms and dot rasters are aligned to stimulus onset (time 0 ). Insets show overlaid individual waveforms of the recorded cells (calibration: $1 \mathrm{~ms}$ ). $\boldsymbol{B}$, Cumulative frequency distributions for PPTg and DA cell response latencies to tone and light.

any effects seen on DA cell activity reflects a specific lateralized action of the PPTg inactivation, rather than a secondary effect on motivation or reduced ability of the animal to perform the task.

The effect of lidocaine infusion is further illustrated in Figure $5 B$, which compares the effect of saline and lidocaine infusion on another DA cell. After several training sessions of cue-solenoid pairing, this cell showed excitatory responses to the tone cue and the light cue, and to the sound of the solenoid when it followed light cues, but had lost solenoid responses when the solenoid was preceded by the tone cue. All of the sensory responses were reduced in amplitude by lidocaine infusion compared with the saline control. Furthermore, in this cell, there appeared to be a weak additional inhibitory response after the tone cue and the solenoid click (but not the light cue), which was also abolished by lidocaine infusion.

The effects illustrated for single cells in Figure 5, $A$ and $B$, were confirmed for the population of DA cells by the quantitative analysis of response amplitudes (Fig. 5C). Statistical analysis showed a significant main effect of treatment (lidocaine vs saline; $F=33.27 ; \mathrm{df}=1,5 ; p=0.002$ ) with no main effect of stimulus type (tone, light, solenoid after tone or solenoid after light; $F=$ 0.646 ; $\mathrm{df}=3,15 ; p=0.598)$ and no significant interaction $(F=$ 2.298 ; $\mathrm{df}=3,15 ; p=0.119)$. Thus, lidocaine infusion significantly reduced the amplitude of activations triggered by all the cues delivered in the task. In contrast, lidocaine had no effect on the baseline firing rate of the cells (calculated from the period in each PSTH before delivery of cues), with mean firing rates of $5.97 \pm 0.9$ and $4.89 \pm 1.1 \mathrm{~Hz}$ in saline and lidocaine tests, respectively $(t=1.43 ; p=0.20 ; n=7$; paired $t$ test).

An additional 22 cells classified as nondopaminergic (firing rates, $0.2-100 \mathrm{~Hz}$; all action potential durations, $<1 \mathrm{~ms}$ ) were selected for testing with PPTg inactivation because they showed some response to cue stimuli or to the click of the solenoid delivering reward. In contrast to the DA group, only six (27\%) of these cells showed changes in firing after PPTg inactivation, and the effect was inconsistent from cell to cell. Two of the cells reduced their baseline firing rate, with no obvious change in sensory response, whereas one cell that responded to conditioned cues showed an increase in responsiveness after lidocaine infusion. In another two cells, there was an increase in the amplitude of activation after the solenoid click, which appeared best related to licking behavior. Only one cell showed a decrease in amplitude of responses to sensory cues. Thus, overall, PPTg inactivation appeared to have a variable effect on nondopaminergic cells in the VTA.

\section{Discussion}

The present data support the hypothesis that PPTg relays sensory signals to evoke phasic activity of DA cells in rewardmediated associative learning. First, PPTg neurons responded to the same task stimuli, both visual and auditory, that activate DA cells. Second, the PPTg responses were dominated by phasic onset patterns, as seen in DA cells. Third, PPTg cells responded earlier than DA cells, allowing for PPTg-to-DA transmission of information. Finally, inactivation of PPTg suppressed the DA cell responses to stimuli, without affecting baseline firing rate.

DA cells are activated by auditory, visual, and somatosensory conditioned stimuli (Romo and Schultz, 1990; Schultz and Romo, 1990). Previous studies have shown that PPTg cells respond to auditory (Reese et al., 1995; Dormont et al., 1998) and somatosensory (Grunwerg et al., 1992) stimuli. The present study confirmed that PPTg cells respond to auditory signals and showed for the first time that they respond to visual stimuli. However, there was a clear bias toward auditory stimuli. Nearly all cells with visual responses also responded to the tone cue; very few showed selectivity for light. This finding contrasts with DA cells, in which, although individual cells can show modality selectivity (as seen in two cases in the present study), there is no evidence for a bias for particular stimuli over the population as a whole (Romo and Schultz, 1990; Schultz and Romo, 1990).

The preference of PPTg for tones raises the possibility that parallel afferent pathways may be involved in regulating DA cell activity, each with a bias for one or other modality. For instance, inputs from superior colliculus (SC) to DA cells have been identified recently (Comoli et al., 2003). SC cells have short latency responses to both light and tone stimuli but with a bias toward visual stimuli (Wallace and Fredens, 1988). We have preliminary evidence in the same paradigm as we used for DA and PPTg cells that a higher proportion of SC cells respond to light (94\%) than to tone (56\%), with only $6 \%$ responding exclusively to tone (M. J. Goddard, W.-X. Pan, B. I. Hyland, unpublished data). Thus, PPTg and SC may play complementary roles in forwarding sensory information of different modalities.

Response patterns in PPTg were also generally consistent with a role in regulating DA cell activity. DA cells show brief bursts of activity at the onset of salient sensory cues, and such phasic "on" responses were the most common in PPTg. However, a sizeable proportion of PPTg cells showed tonic components, which are 

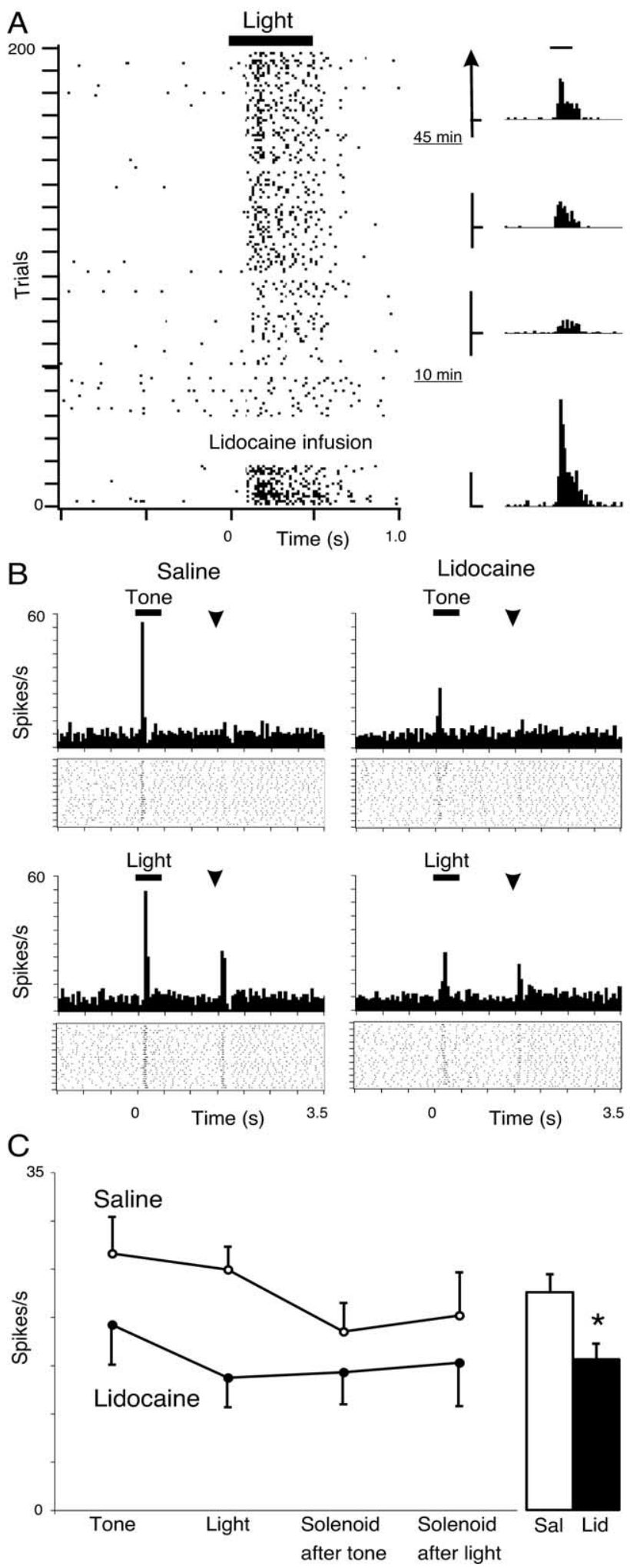

Figure 5. Effect of PPTg inactivation on responses of DA cells. $A$, Time course of the lidocaine infusion effect. The dot raster shows the response of a DA cell to a light cue (onset at time 0 ) on individual trials before and after infusion of lidocaine to the PPTg. The trials are arranged in order from bottom to top. The histograms show activity averaged over trials indicated by the vertical bars, before lidocaine infusion (bottom) and for three periods after infusion (full vertical calibration: 70 spikes/s on all histograms). Light-cue duration ( $0.5 \mathrm{~s}$ ) is indicated by the horizontal bar above the dot raster and the histograms. This cell did not respond to the tone cue on randomly intermingled tone trials, which are not included in the figure. $\boldsymbol{B}$, Comparison of lidocaine and saline infusion. Histograms and dot rasters show activity of another DA cell in not common in DA cells, although they can be seen in certain circumstances (Fiorillo et al., 2003). Another unusual feature of PPTg activity was the occurrence of pure "off" responses at the termination of cues. These have not been reported in DA cells, and whether they could have some role in regulating DA cell activity or reflect activity in PPTg neurons projecting to other targets remains to be established.

The present experiment showed that PPTg neurons respond to auditory stimuli at extremely short latency, tightly time locked to the stimulus. These ultra-short latencies are similar to those reported previously for PPTg cells in cats (Reese et al., 1995; Dormont et al., 1998) and suggest relative direct access to PPTg from structures early in the auditory pathway. Efferents from PPTg are therefore well placed to provide accurate information about the timing of sensory signals, necessary for building associations between sensory events.

Importantly, the average PPTg latencies for auditory and visual cues were significantly shorter than for DA cells, consistent with a PPTg-to-DA transmission of information. This is supported by the fact that the difference in latency of stimulus-locked PPTg and DA burst responses we found is similar to the latency of bursts induced in DA cells by PPTg stimulation in anesthetized rats (Lokwan et al., 1999). The latency of these bursts is longer than might be expected from activity in the direct projections from PPTg to the DA cell fields (Scarnati et al., 1984; Beninato and Spencer, 1987; Bolam et al., 1991; Futami et al., 1995; Lokwan et al., 1999), which give rise to monosynaptic EPSPs and singlespike responses in DA cells at onset latencies of $<10 \mathrm{~ms}$ (Scarnati et al., 1984; Futami et al., 1995). Burst responses may thus reflect activity in polysynaptic pathways from PPTg. Alternatively, it has been suggested that biophysical properties of DA cells may lead to a delay in onset of bursts in response to monosynaptic inputs (Overton and Clark, 1997; Lokwan et al., 1999).

The most direct evidence for involvement of PPTg in regulating DA cell function was obtained from the PPTg inactivation experiments. Lidocaine injection into PPTg consistently decreased responses of DA cells to conditioned stimuli. Responses were never completely abolished, which may reflect incomplete inactivation of the PPTg by the infusion, or, as discussed above, the presence of other inputs from structures such as SC that might be able to partly sustain DA cell activity. On the other hand, it was notable that despite the numerical bias for auditory responses in PPTg neuronal activity, there were similar effects on light and tone cue responses of DA cells after PPTg inactivation, suggesting that the visual component of PPTg responses has a functionally important role.

Importantly, inactivation of PPTg specifically reduced DA cell responses to cue signals, without, for the number of cells available, a significant effect on their baseline firing rate. This is consistent with previous findings that PPTg selectively controls the

$\leftarrow$

response to tone (top), light (bottom), and solenoid click (arrowhead; after either tone or light) stimuli. The left panels show postsaline control data, and the right panels show responses of the same cell after lidocaine. The histograms were constructed from the period 5-25 min after infusion. C, Quantitative analysis of the effect of lidocaine infusion to PPTg on DA cell activity. The interaction plot shows mean firing rate in the poststimulus period across all cells for different stimulus types (tone and light cues, solenoid after tone, solenoid after light) separately for saline (open circles) and lidocaine (filled circles) infusion experiments. The bar graph shows the mean poststimulus firing rate for saline (Sal; open bar) and lidocaine (Lid; filled bar) experiments averaged across stimulus type. There was a significant main effect for saline versus lidocaine but no significant effect for stimulus type and no significant interaction. Error bars represent SEM. ${ }^{*} p<0.01$. 
bursting of DA cells rather than their tonic resting activity (Floresco et al., 2003). Together with consideration of latencies for burst responses discussed above, these data support models in which PPTg inputs have a particularly important role in generating burst responses of DA cells to salient sensory stimuli (Schultz, 1998; Brown et al., 1999).

Whether PPTg has a role beyond the simple relay of accurately timed sensory information remains enigmatic. Previous studies have shown that integrity of PPTg is necessary for arousal, attention, motivation and learning, and, particularly interestingly here, stimulus-reward associations (Steckler et al., 1994b). Studies using a variety of conditioning tasks have suggested a role for PPTg in assigning task-specific salience value to cue signals (Inglis et al., 1994, 2000; Florio et al., 1999; Kippin and van der Kooy, 2003). Consistent with this idea, some PPTg cells in cat were found to respond to conditioned stimuli in a context-dependent way, failing to respond to the same cue when another signal indicated a different task requirement (Dormont et al., 1998). However, these data are difficult to interpret, because there were multiple sequential sensory cues, which may have triggered prepulse inhibition of later responses (Bickford et al., 1993; Moxon et al., 1999).

The present study provides only limited support for the idea that PPTg neuronal activity may code context. The great majority of PPTg cells responded to sensory stimuli, whether or not they were associated with rewards, consistent with findings that lesions of PPTg do not disrupt reward value of brain stimulation (Waraczynski and Perkins, 1998) or sucrose (Keating et al., 2002). Only two PPTg cells showed plasticity of responses to stimuli depending on the association of the stimuli with rewards, suggesting that PPTg contributes relatively little to integration of information about reward context. Consistent with this, although lesions of PPTg do affect conditioning of tasks with specific sensory cues (Inglis et al., 2000), they do not impair the learning of tasks such as place preference (Bechara and van der Kooy, 1989) or delayed nonmatching to position (Steckler et al., 1994a), in which context of signals is important.

Although PPTg might not be primarily involved in processing of reward context, it may have a role in attentional mechanisms. Lesions of PPTg specifically impair global attention, and this effect could account for results previously interpreted as reflecting difficulties with associative learning (Inglis et al., 2001). Recent data show that PPTg cells respond less to poorly attended cues (Ivlieva and Timofeeva, 2003), suggesting that an attentional or arousal gate acts at this level. Together with the present data, this leads to models in which PPTg acts as an interface between primary sensory and motivational systems, providing information about signals to which the animal is attending, regardless of rewardvalue (see supplementalmaterial, available atwww.jneurosci. org). That DA cells generally respond best only when such signals are associated with reward, whereas PPTg cells respond noncontingently, suggests that the PPTg inputs may be gated by separate, reward-information-bearing pathways.

In summary, the present results confirm a central role for PPTg in determining the reward-mediated learning activity of DA cells. The evidence is most consistent with PPTg serving as an interface between primary sensory pathways and DA cells. We propose that the PPTg input may have dual role, providing accurate, short latency information about the timing of sensory stimuli and a higher-level function in conveying information specifically about stimuli that have been passed by sensory-attention gating mechanisms.

\section{References}

Aebischer P, Schultz W (1984) The activity of pars compacta neurons of the monkey substantia nigra is depressed by apomorphine. Neurosci Lett 50:25-29.

Aghajanian GK, Bunney BS (1977) Dopamine "autoreceptors": pharmacological characterization by microiontophoretic single cell recording studies. Naunyn Schmiedebergs Arch Pharmacol 297:1-7.

Bechara A, van der Kooy D (1989) The tegmental pedunculopontine nucleus: a brain-stem output of the limbic system critical for the conditioned place preferences produced by morphine and amphetamine. J Neurosci 9:3400-3409.

Beninato M, Spencer RF (1987) A cholinergic projection to the rat substantia nigra from the pedunculopontine tegmental nucleus. Brain Res 412:169-174.

Bickford PC, Luntz-Leybman V, Freedman R (1993) Auditory sensory gating in the rat hippocampus: modulation by brainstem activity. Brain Res 607:33-38.

Blackburn JR, Pfaus JG, Phillips AG (1992) Dopamine functions in appetitive and defensive behaviours. Prog Neurobiol 39:247-279.

Bolam JP, Francis CM, Henderson Z (1991) Cholinergic input to dopaminergic neurons in the substantia nigra: a double immunocytochemical study. Neuroscience 41:483-494.

Brown J, Bullock D, Grossberg S (1999) How the basal ganglia use parallel excitatory and inhibitory learning pathways to selectively respond to unexpected rewarding cues. J Neurosci 19:10502-10511.

Bunney BS, Aghajanian GK, Roth RH (1973) Comparison of effects of L-dopa, amphetamine and apomorphine on firing rate of rat dopaminergic neurones. Nat New Biol 245:123-125.

Comoli E, Coizet V, Boyes J, Bolam JP, Canteras NS, Quirk RH, Overton PG, Redgrave P (2003) A direct projection from superior colliculus to substantia nigra for detecting salient visual events. Nat Neurosci 6:974-980.

Diana M, Tepper JM (2002) Electrophysiological pharmacology of mesencephalic dopaminergic neurons. In: Dopamine in the CNS II (Di Chiara G, ed), pp 1-61. Berlin: Springer.

Dormont JF, Conde H, Farin D (1998) The role of the pedunculopontine tegmental nucleus in relation to conditioned motor performance in the cat I. Context-dependent and reinforcement-related single unit activity. Exp Brain Res 121:401-410.

Fiorillo CD, Tobler PN, Schultz W (2003) Discrete coding of reward probability and uncertainty by dopamine neurons. Science 299:1898-1902.

Floresco SB, West AR, Ash B, Moore H, Grace AA (2003) Afferent modulation of dopamine neuron firing differentially regulates tonic and phasic dopamine transmission. Nat Neurosci 6:968-973.

Florio T, Capozzo A, Puglielli E, Pupillo R, Pizzuti G, Scarnati E (1999) The function of the pedunculopontine nucleus in the preparation and execution of an externally-cued bar pressing task in the rat. Behav Brain Res 104:95-104.

Forster GL, Blaha CD (2003) Pedunculopontine tegmental stimulation evokes striatal dopamine efflux by activation of acetylcholine and glutamate receptors in the midbrain and pons of the rat. Eur J Neurosci 17:751-762.

Futami T, Takakusaki K, Kitai ST (1995) Glutamatergic and cholinergic inputs from the pedunculopontine tegmental nucleus to dopamine neurons in the substantia nigra pars compacta. Neurosci Res 21:331-342.

Grace AA, Bunney BS (1980) Nigral dopamine neurons: intracellular recording and identification with L-dopa injection and histofluorescence. Science 210:654-656.

Grace AA, Bunney BS (1983) Intracellular and extracellular electrophysiology of nigral dopaminergic neurons-1. Identification and characterization. Neuroscience 10:301-315.

Grunwerg BS, Krein H, Krauthamer GM (1992) Somatosensory input and thalamic projection of pedunculopontine tegmental neurons. NeuroReport 3:673-675.

Hyland BI, Reynolds JNJ, Hay J, Perk CG, Miller R (2002) Firing modes of midbrain dopamine cells in the freely moving rat. Neuroscience 114:475-492.

Inglis WL, Dunbar JS, Winn P (1994) Outflow from the nucleus accumbens to the pedunculopontine tegmental nucleus: a dissociation between locomotor activity and the acquisition of responding for conditioned reinforcement stimulated by D-amphetamine. Neuroscience 62:51-64.

Inglis WL, Olmstead MC, Robbins TW (2000) Pedunculopontine tegmen- 
tal nucleus lesions impair stimulus-reward learning in autoshaping and conditioned reinforcement paradigms. Behav Neurosci 114:285-294.

Inglis WL, Olmstead MC, Robbins TW (2001) Selective deficits in attentional performance on the 5-choice serial reaction time task following pedunculopontine tegmental nucleus lesions. Behav Brain Res 123:117-131.

Ivlieva NY, Timofeeva NO (2003) Activity of neurons in the pedunculopontine nucleus during a food-related operant conditioned reflex. Neurosci Behav Physiol 33:919-928.

Keating GL, Walker SC, Winn P (2002) An examination of the effects of bilateral excitotoxic lesions of the pedunculopontine tegmental nucleus on responding to sucrose reward. Behav Brain Res 134:217-228.

Kippin TE, van der Kooy D (2003) Excitotoxic lesions of the tegmental pedunculopontine nucleus impair copulation in naive male rats and block the rewarding effects of copulation in experienced male rats. Eur J Neurosci 18:2581-2591.

Ljungberg T, Apicella P, Schultz W (1992) Responses of monkey dopamine neurons during learning of behavioral reactions. J Neurophysiol 67:145-163.

Lokwan SJ, Overton PG, Berry MS, Clark D (1999) Stimulation of the pedunculopontine tegmental nucleus in the rat produces burst firing in A9 dopaminergic neurons. Neuroscience 92:245-254.

Miller JD, Sanghera MK, German DC (1981) Mesencephalic dopaminergic unit activity in the behaviorally conditioned rat. Life Sci 29:1255-1263.

Mirenowicz J, Schultz W (1996) Preferential activation of midbrain dopamine neurons by appetitive rather than aversive stimuli. Nature 379:449-451.

Moxon KA, Gerhardt GA, Bickford PC, Austin K, Rose GM, Woodward DJ, Adler LE (1999) Multiple single units and population responses during inhibitory gating of hippocampal auditory response in freely-moving rats. Brain Res 825:75-85.

Overton PG, Clark D (1997) Burst firing in midbrain dopaminergic neurons. Brain Res Brain Res Rev 25:312-334.
Paxinos G, Watson C (1997) The rat brain in stereotaxic coordinates, Ed 3. London: Academic.

Reese NB, Garcia-Rill E, Skinner RD (1995) Auditory input to the pedunculopontine nucleus: II. Unit responses. Brain Res Bull 37:265-273.

Romo R, Schultz W (1990) Dopamine neurons of the monkey midbrain: contingencies of responses to active touch during self-initiated arm movements. J Neurophysiol 63:592-606.

Scarnati E, Campana E, Pacitti C (1984) Pedunculopontine-evoked excitation of substantia nigra neurons in the rat. Brain Res 304:351-361.

Schultz W (1986) Responses of midbrain dopamine neurons to behavioral trigger stimuli in the monkey. J Neurophysiol 56:1439-1461.

Schultz W (1998) Predictive reward signal of dopamine neurons. J Neurophysiol 80:1-27.

Schultz W, Romo R (1990) Dopamine neurons of the monkey midbrain: contingencies of responses to stimuli eliciting immediate behavioral reactions. J Neurophysiol 63:607-624.

Steckler T, Keith AB, Sahgal A (1994a) Lesions of the pedunculopontine tegmental nucleus do not alter delayed non-matching to position accuracy. Behav Brain Res 61:107-112.

Steckler T, Inglis W, Winn P, Sahgal A (1994b) The pedunculopontine tegmental nucleus: a role in cognitive processes? Brain Res Brain Res Rev 19:298-318.

Waelti P, Dickinson A, Schultz W (2001) Dopamine responses comply with basic assumptions of formal learning theory. Nature 412:43-48.

Wallace MN, Fredens K (1988) Origin of high acetylcholinesterase activity in the mouse superior colliculus. Exp Brain Res 72:335-346.

Waraczynski M, Perkins M (1998) Lesions of pontomesencephalic cholinergic nuclei do not substantially disrupt the reward value of medial forebrain bundle stimulation. Brain Res 800:154-169.

Wise RA (2002) Brain reward circuitry: insights from unsensed incentives. Neuron 36:229-240. 